Supporting Information

\title{
The Proximal Lilly Collection: Mapping, Exploring and Exploiting Feasible Chemical Space
}

\author{
AUTHOR NAMES: Christos A. Nicolaou*, Ian A. Watson, Hong Hu, Jibo Wang \\ AUTHOR ADDRESS: Discovery Chemistry, Lilly Research Laboratories, Eli Lilly and \\ Company, Indianapolis, IN 46285
}

Corresponding author email: c.nicolaou@lilly.com 
Table S1: Pubchem IDs for diphenpyramide and 11 compounds screened for RIO2 kinase binding affinity.

\begin{tabular}{|c|l|}
\hline Diphenpyramide & PBCHM24238955 \\
\hline 1 & PBCHM4744022 \\
\hline 3 & PBCHM17399864 \\
\hline 2 & PBCHM70116143 \\
\hline 7 & PBCHM24238955 \\
\hline 6 & PBCHM17249003 \\
\hline 5 & PBCHM4680899 \\
\hline 4 & PBCHM58447945 \\
\hline 8 & PBCHM17395068 \\
\hline 9 & PBCHM25348574 \\
\hline 10 & PBCHM35023250 \\
\hline 11 & PBCHM25336050 \\
\hline
\end{tabular}


Table S2: The 10 main LARR reactions

Note 1: A comprehensive description of the Lilly substructure search engine used for annotating PLC reagents can be found in the Suporting Information of the paper:

Bruns, R. F.; Watson, I. A., Rules for Identifying Potentially Reactive or Promiscuous Compounds. Journal of medicinal chemistry 2012, 55 (22), 9763-9772.

Note 2: Legend for Lilly custom smarts extensions implementation symbols:

T: number of attached heteroatoms to an atom

IIw: exclude following atom from the matched atoms reported back to the calling program

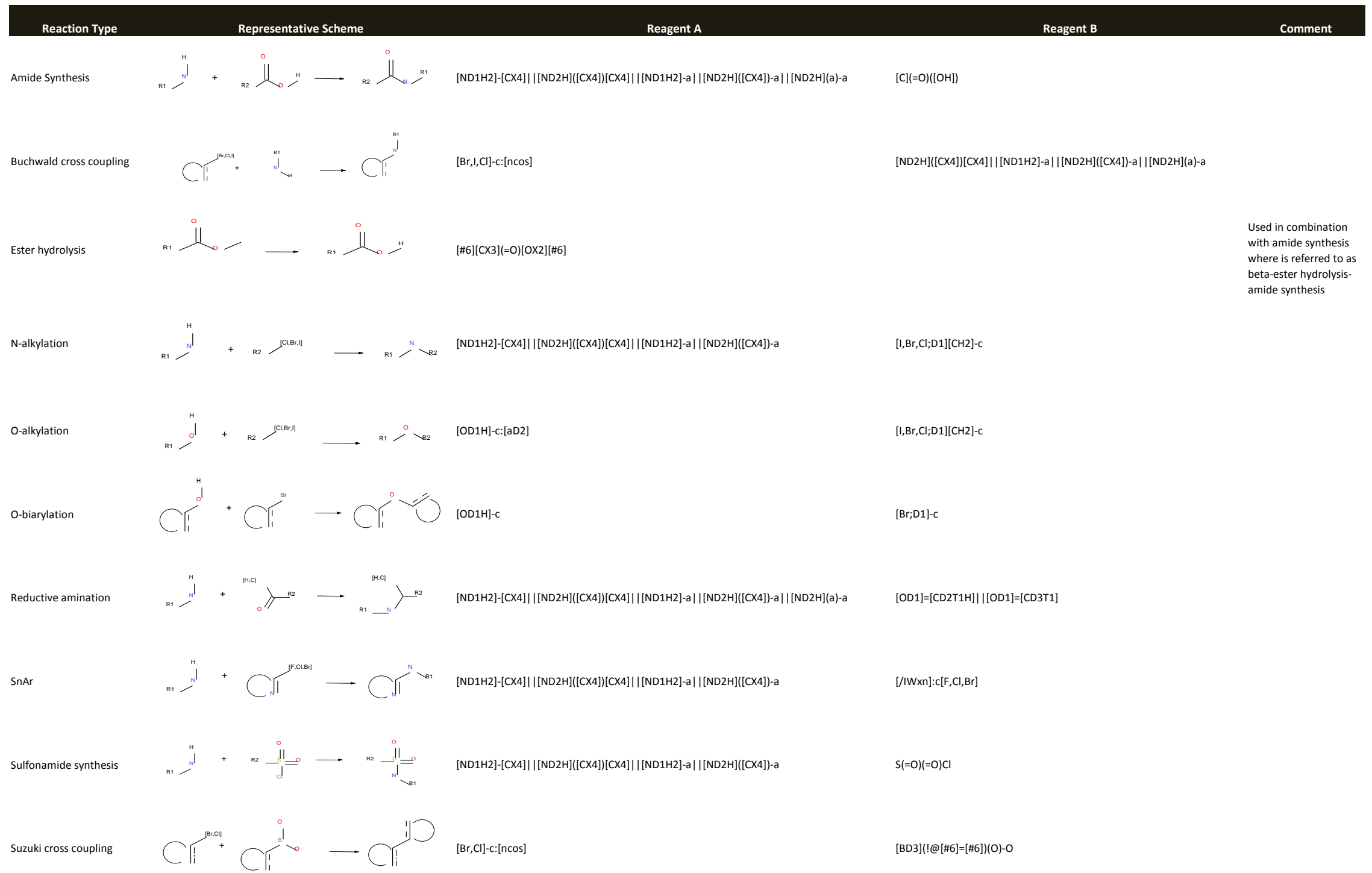


Table S3: Annotations for each of the LARR reactions reagent types

Note: The annotations below are calculated using the definitions in Table S4.

Reaction: Amide synthesis; Reagent: Carboxylic acids

carboxylic_acids $=1$

acetal $=0$

acid_chlorides $=0$

alcohols $=0$

aldehydes $=0$

alkynes $=0$

amidines $=0$

amines_aliphatic_primary $=0$

amines_aliphatic_secondary $=0$

amines_aromatic_primary $=0$

amines_aromatic_secondary_1 $=0$

amines_aromatic_secondary_2 $=0$

oxime $=0$

boronic_acids $=0$

chloroformates $=0$

diols $=0$

guanidines $=0$

halides_alkyl $=0$

hydrazines $=0$

imines $=0$

isocyanates $=0$

isocyanides $=0$

isothiocyanates $=0$

nhydroxyamides $=0$

oxime $=0$

phenols $=0$

sulfonic_acids $=0$

sulfonyl_chlorides $=\odot$

thiols $=0$

isotope $=0$

Phosphorus $=0$

nonorganic $=0$

fragments $=0$

Reaction: Amide synthesis; Reagent: Amines

amines_aliphatic_primary + amines_aliphatic_secondary +

amines_aromatic_primary + amines_aromatic_secondary_1 +

amines_aromatic_secondary_2 = 1

acetal $=0$

acid_chlorides $=0$

aldehydes $=0$

alcohols $=0$

alkynes $=0$

amidines $=0$

boronic_acids $=0$

carboxylic_acids $=0$

chloroformates $=0$

diols $=0$ 


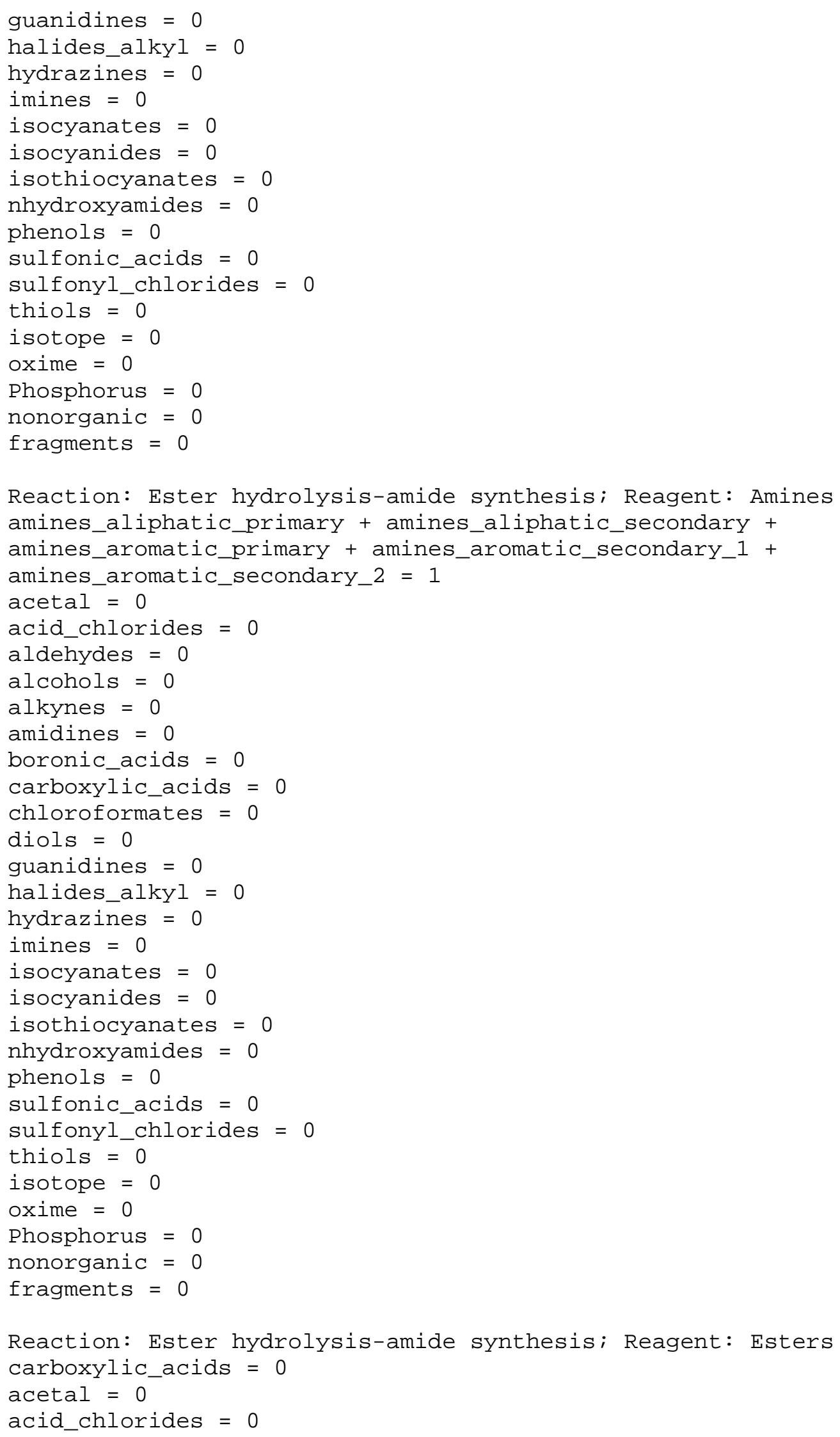

Reaction: Ester hydrolysis-amide synthesis; Reagent: Esters carboxylic_acids $=0$

acetal $=0$

acid_chlorides $=0$ 


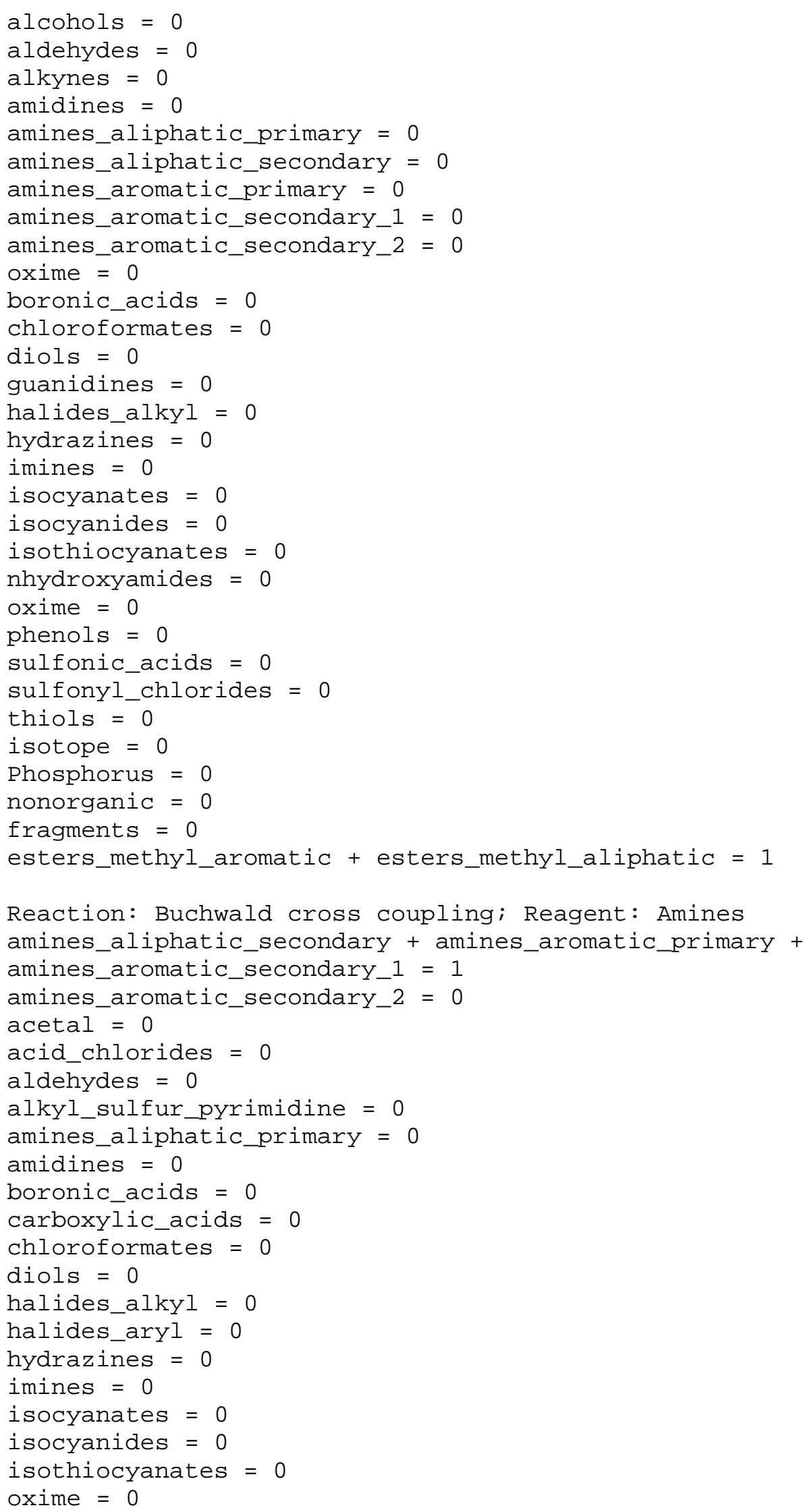




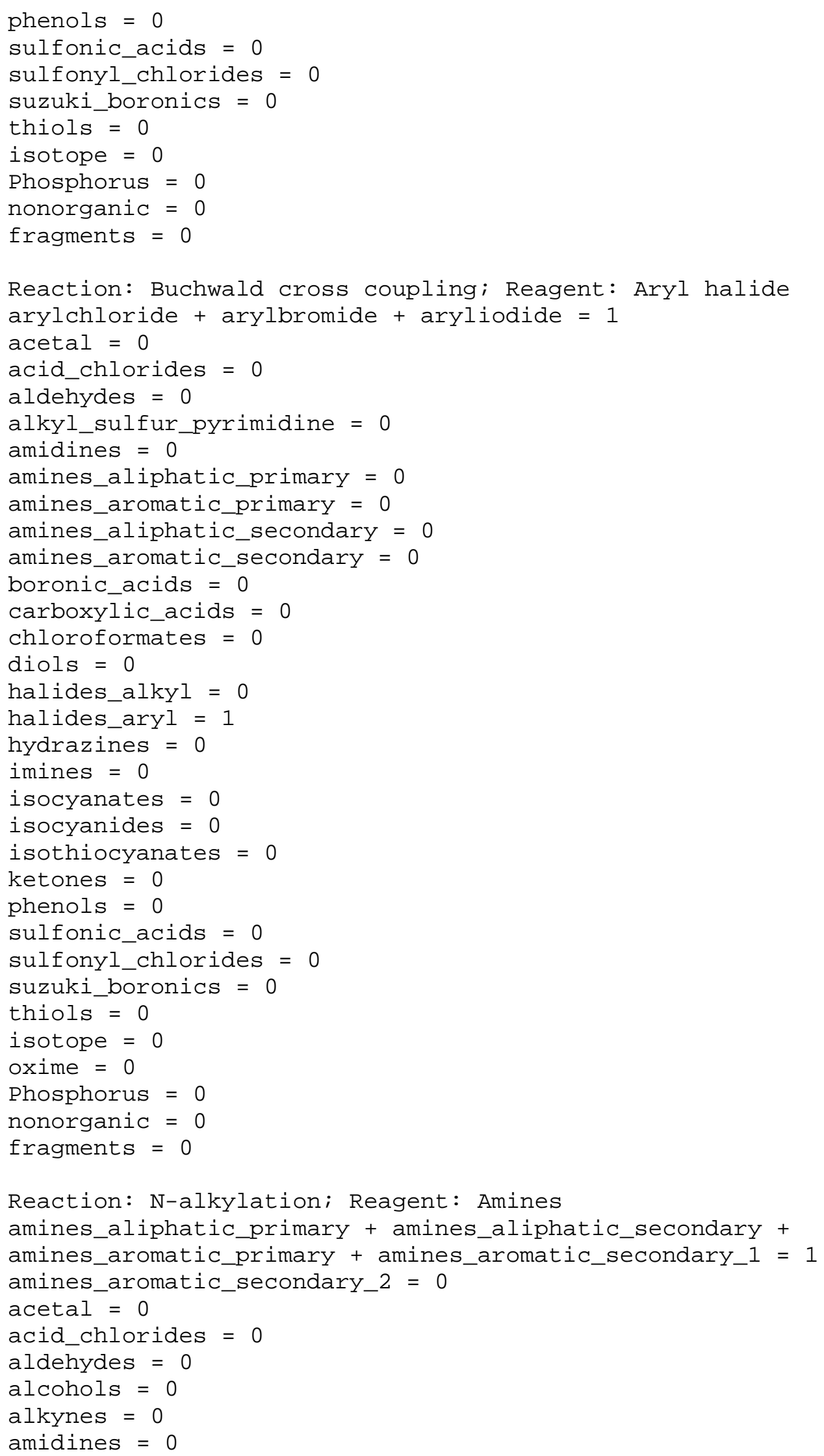




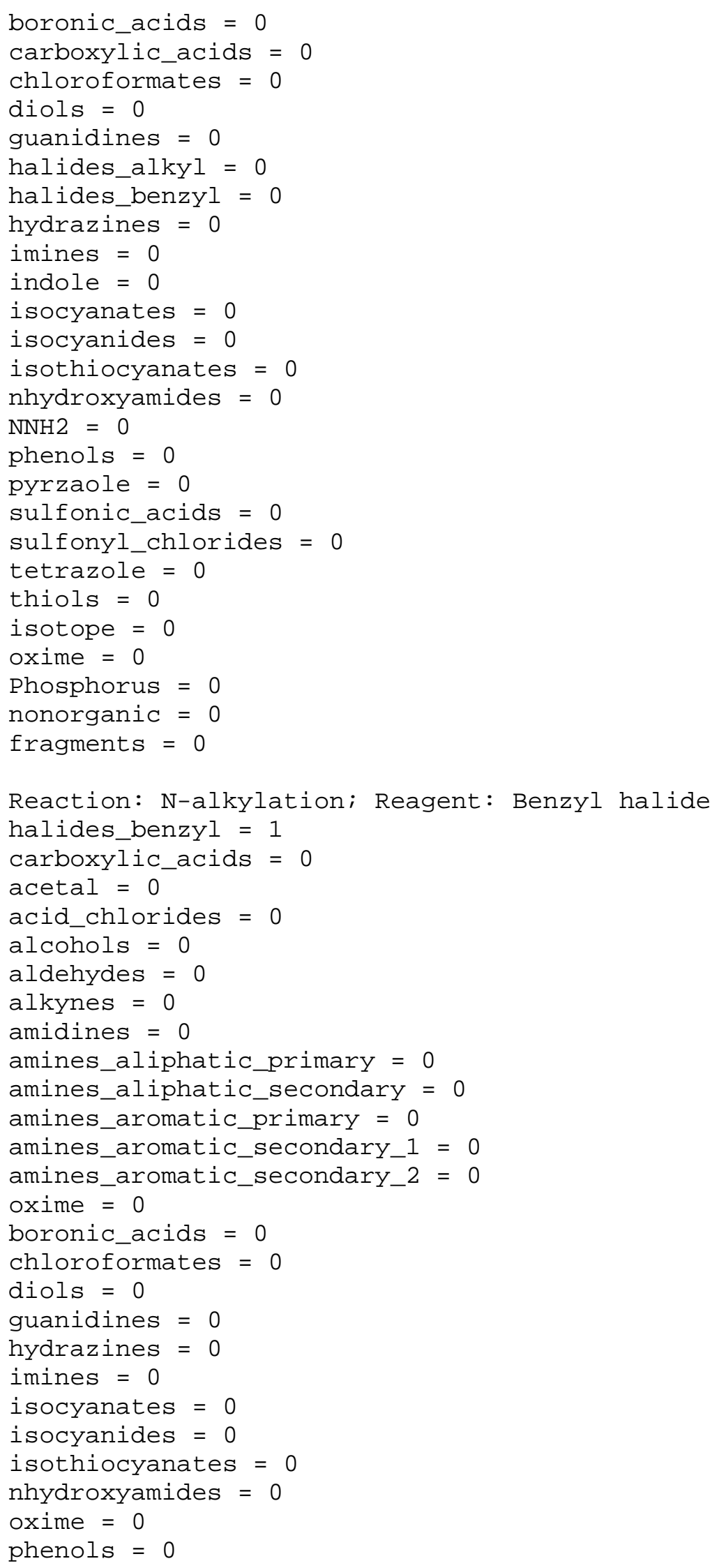




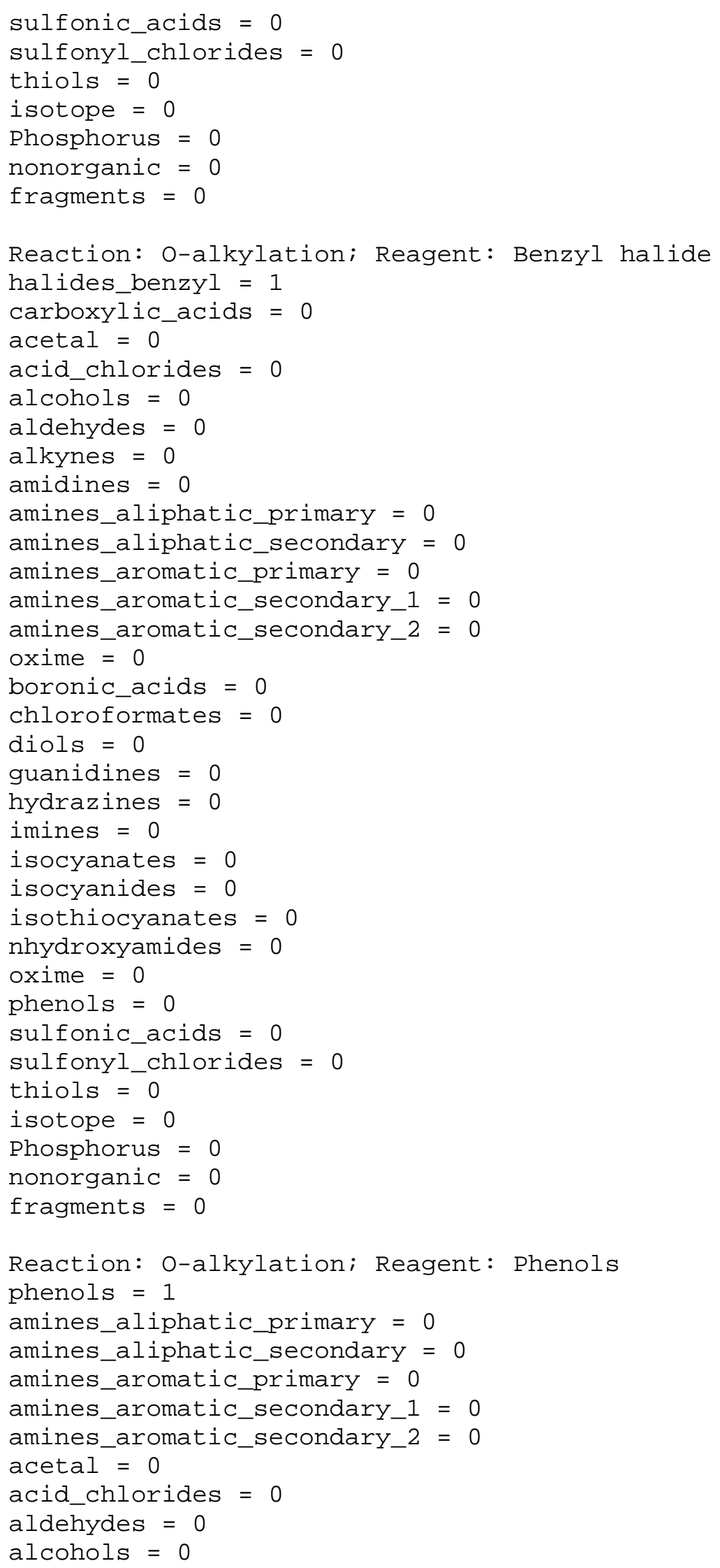

Reaction: 0-alkylation; Reagent: Phenols phenols $=1$ 


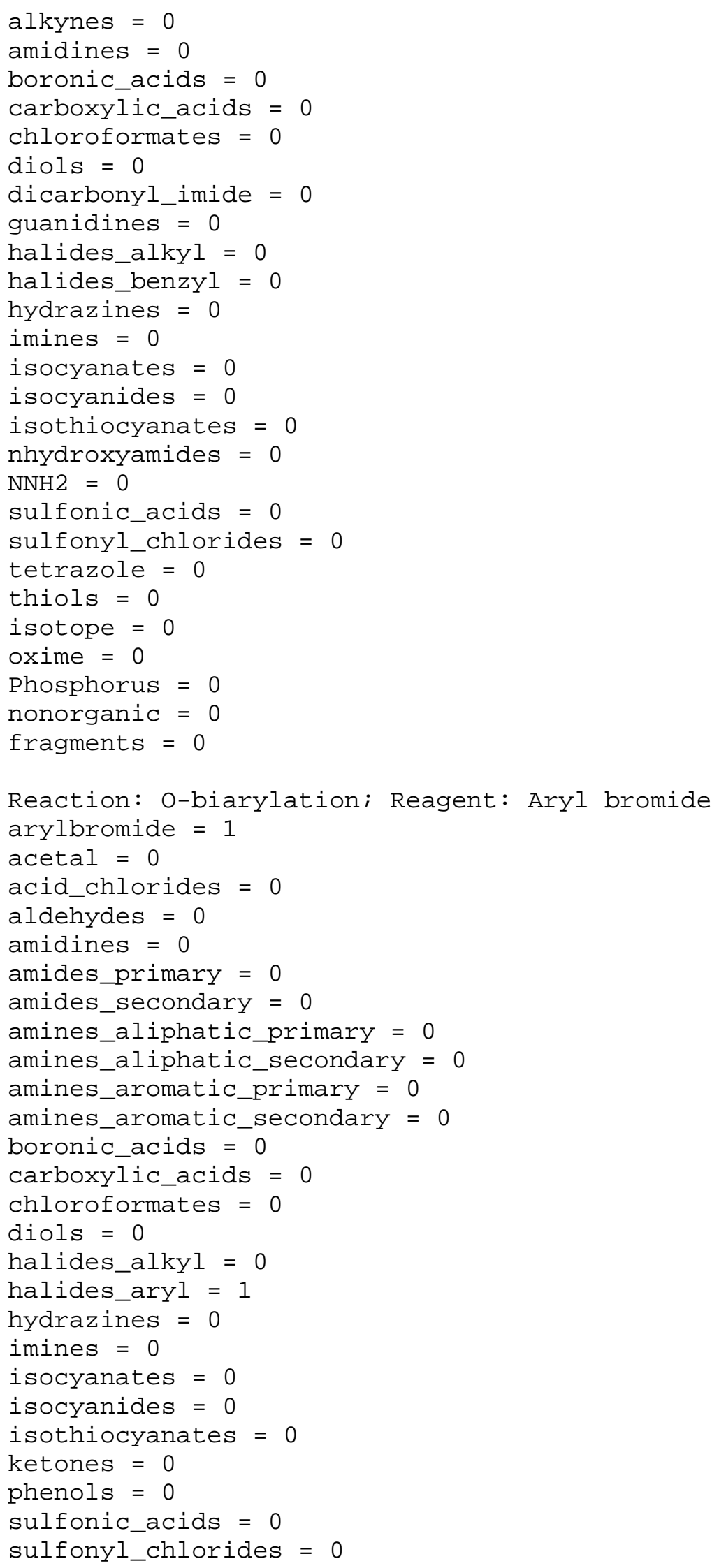




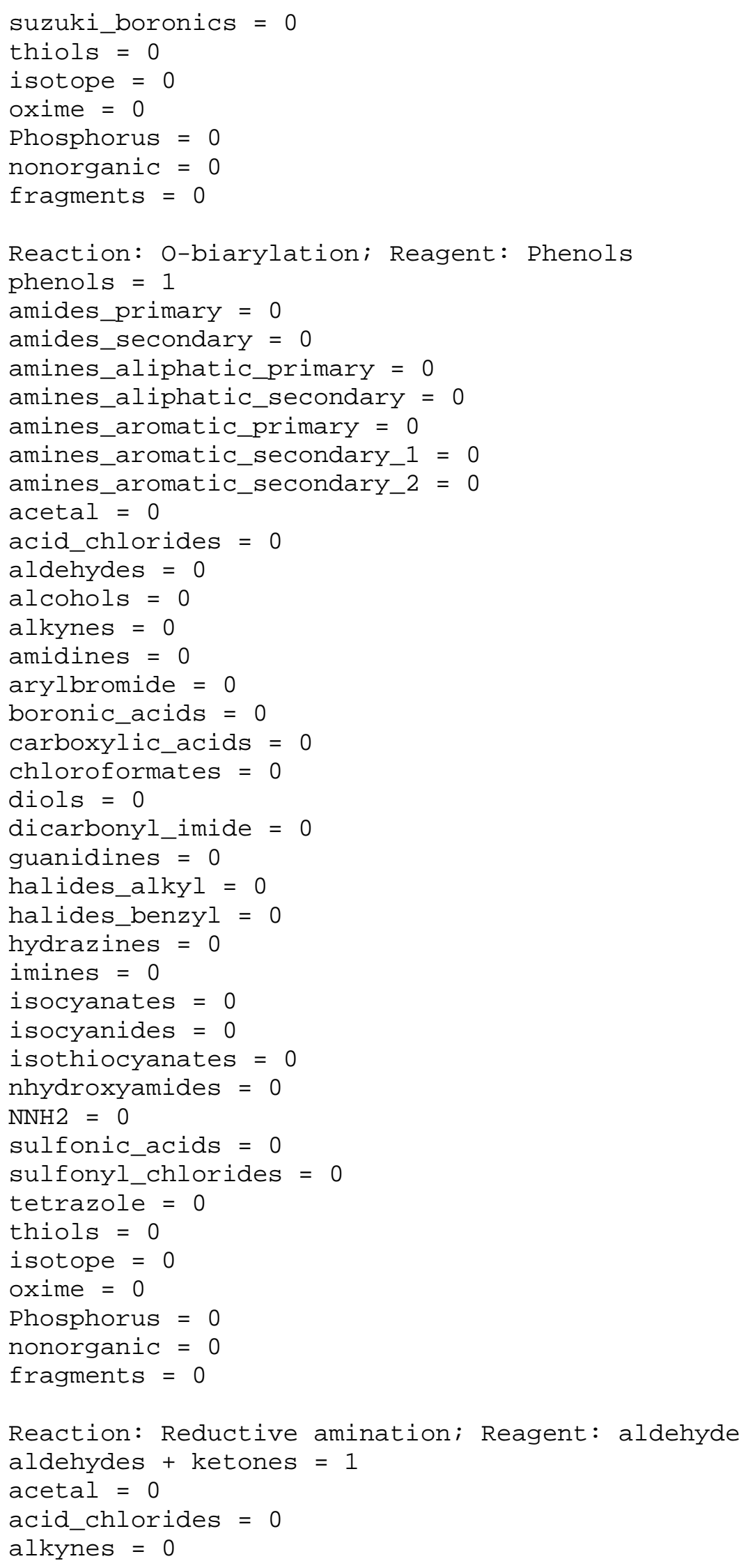

Reaction: Reductive amination; Reagent: aldehyde aldehydes + ketones $=1$ acetal $=0$ acid_chlorides $=0$ alkynes $=0$ 


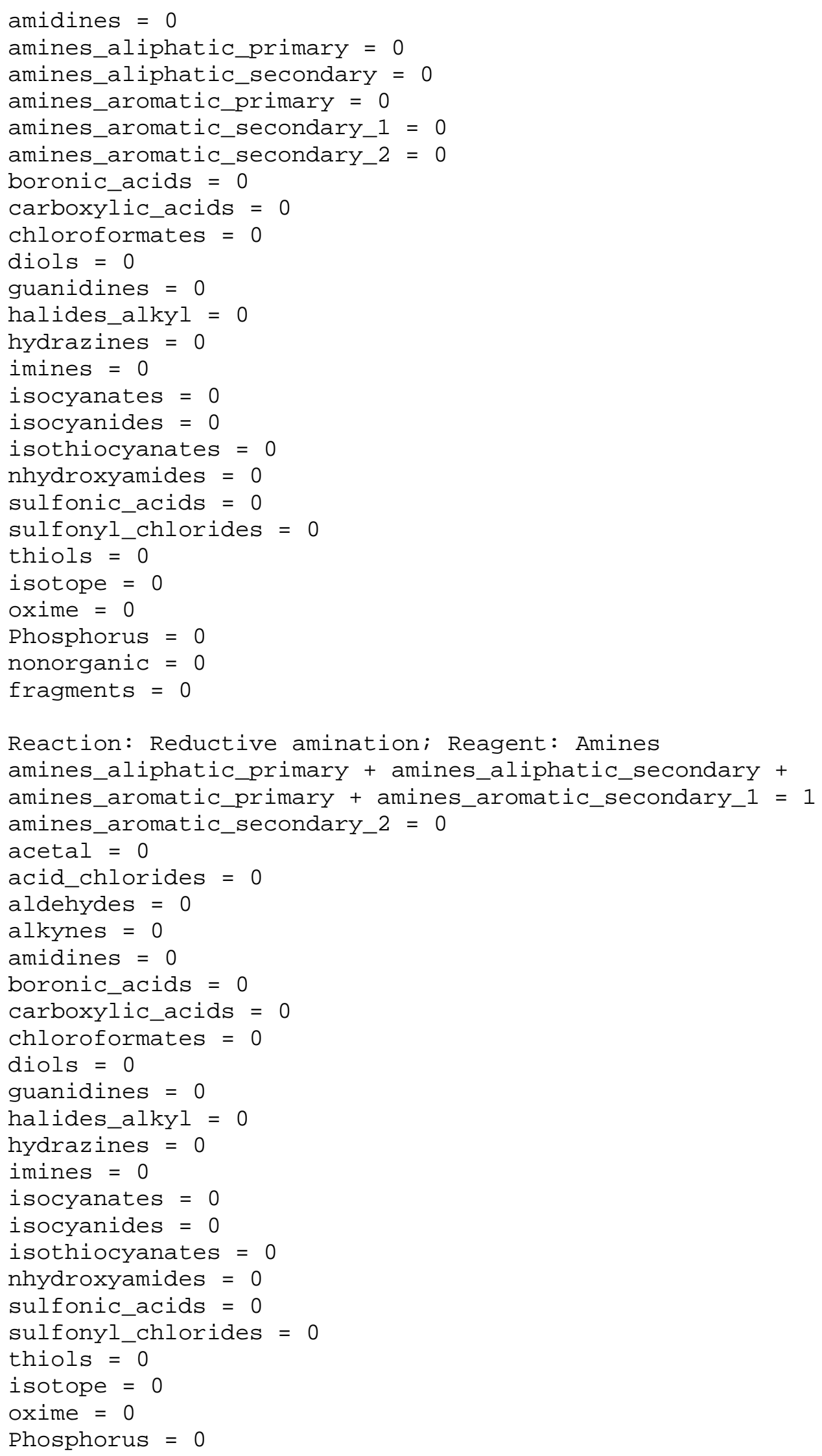




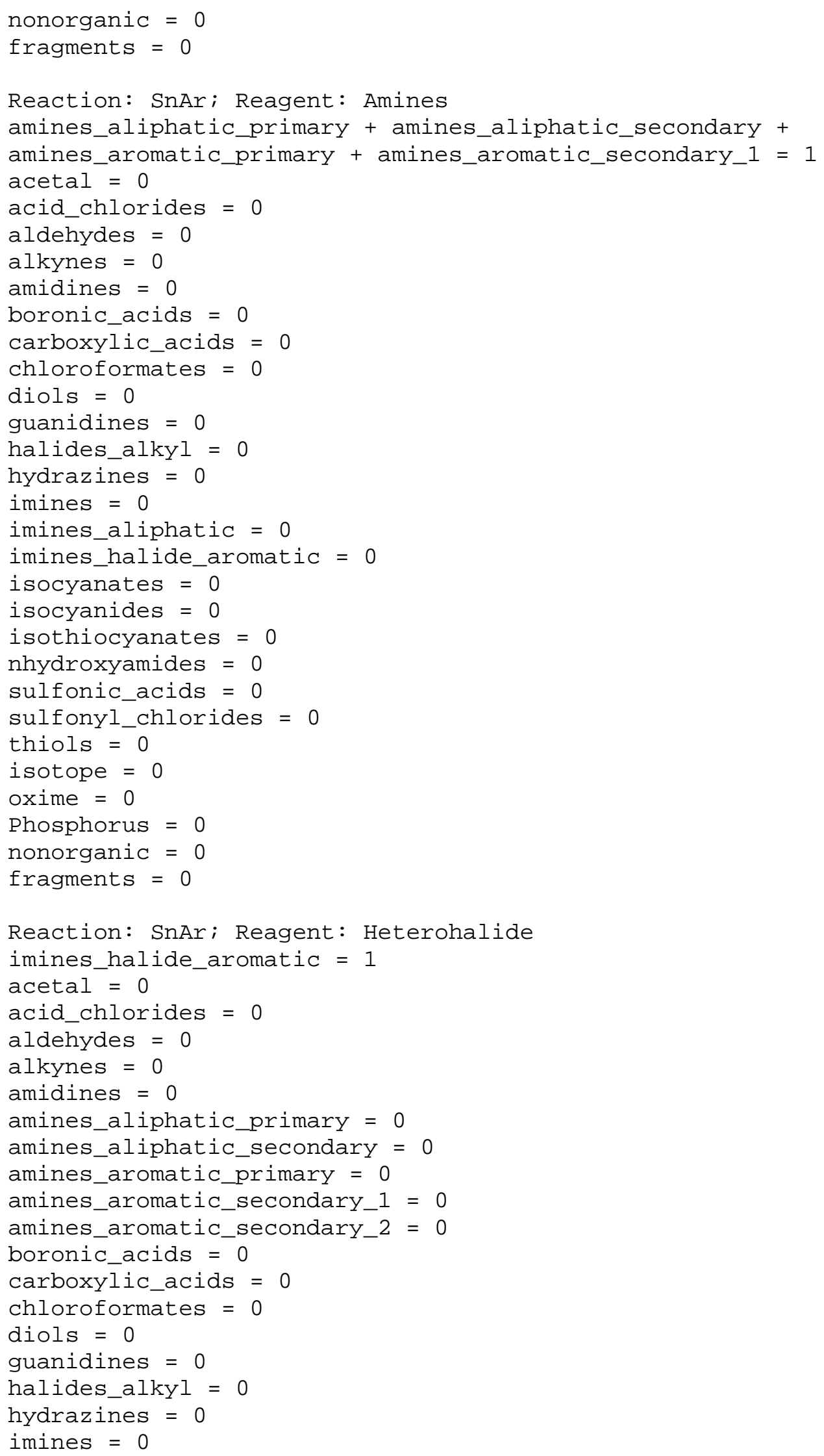




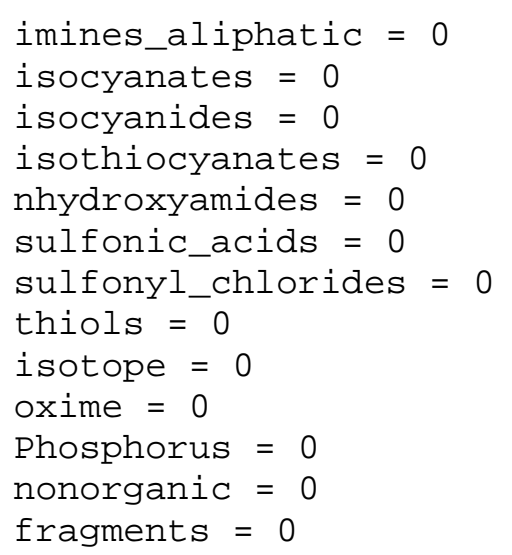

Reaction: Sulfonamide synthesis; Reagent: Amines amines_aliphatic_primary + amines_aliphatic_secondary + amines_aromatic_primary + amines_aromatic_secondary_1 $=1$

Reaction: Sulfonamide synthesis; Reagent: Sulfonil chlorides sulfonyl_chlorides $=1$ acetal $=0$ 


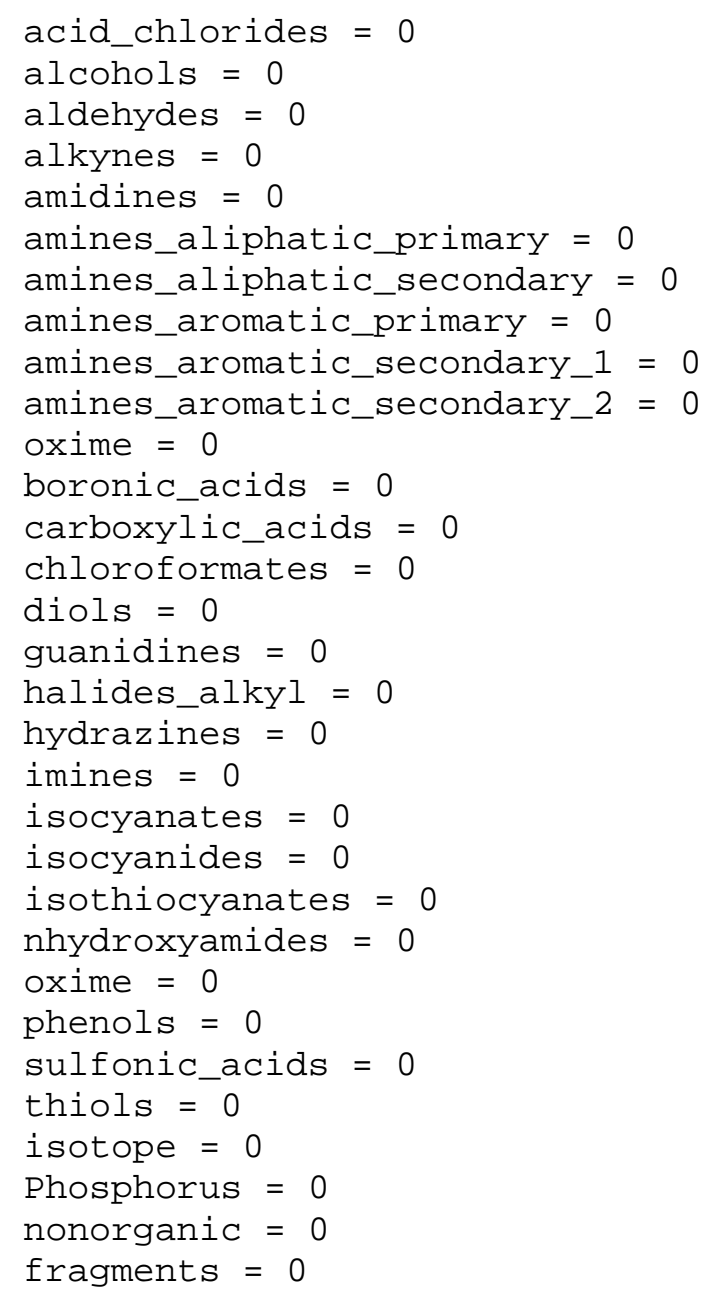

Reaction: Suzuki crosss coupling; Reagent: Aryl bromide arylchloride + arylbromide $=1$

acetal $=0$

acid_chlorides $=0$

aldehydes $=0$

amidines $=0$

amines_aliphatic_primary $=0$

amines_aromatic_primary $=0$

boronic_acids $=0$

carboxylic_acids $=0$

chloroformates $=0$

diols $=0$

halides_alkyl $=0$

halides_aryl $=1$

hydrazines $=0$

imines $=0$

isocyanates $=0$

isocyanides $=0$

isothiocyanates $=0$

ketones $=0$

phenols $=0$

sulfonic_acids $=0$ 


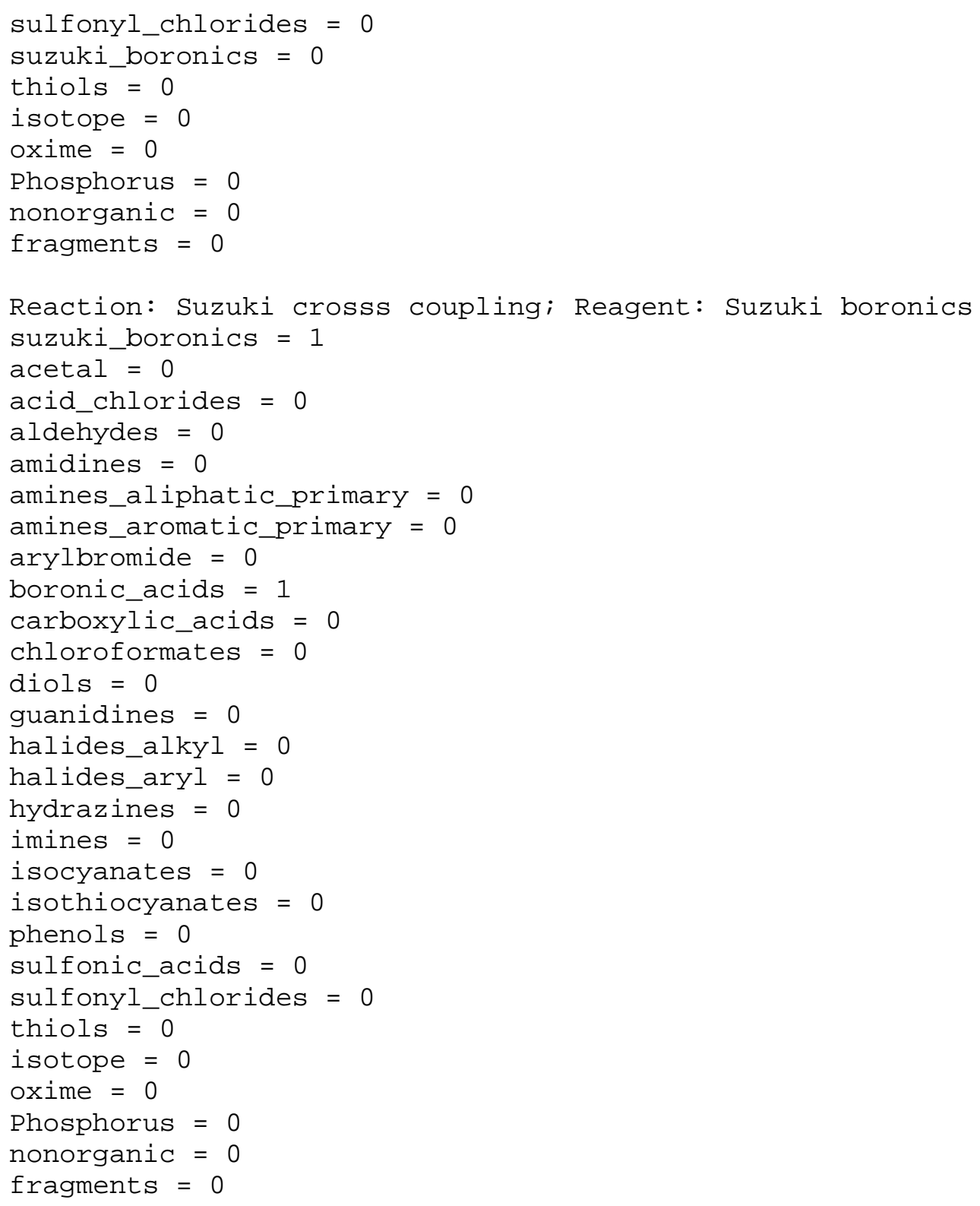


Table S4: Queries for PLC reagent annotations

Note 1: A comprehensive description of the Lilly substructure search engine used for annotating PLC reagents can be found in the supporting Information of the paper:

Bruns, R. F.; Watson, I. A., Rules for Identifying Potentially

Reactive or Promiscuous Compounds. Journal of medicinal chemistry 2012, 55 (22), 9763-9772.

Note 2: Legend for Lilly custom smarts extensions implementation symbols:

$T \quad$ number of attached heteroatoms to an atom

G number of unsaturations associated with an atom

IIwX exclude following atom from the matched atoms reported back to the calling program

Queries (in alphabetical order):

( $\odot$ Query

(A I version 2)

(A C Comment "acetal")

)

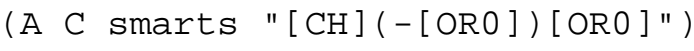

( $\odot$ Query

(A I version 2)

(A C Comment "acid_chlorides")

)

(A C smarts "Cl-[CT2]=0")

( $\odot$ Query

(A I version 2)

(A C Comment "alcohols")

)

(A C smarts "[OD1H]-[CGOT1]")

( $\odot$ Query

(A I Version 2)

(A C Comment "aldehydes")

)

(A C smarts "[OD1]=[CD2T1H]-*")

( $\odot$ Query

(A C Comment "alkyl_sulfur_pyrimidine")

(A I Version 2)

)

(A C smarts "c $(n)(n) S[C X 4] "$ )

( 0 Query

(A I version 2)

(A C Comment "alkynes")

)

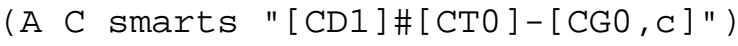

( $\odot$ query

(A I version 2)

(A C Comment "amides_alphatic") 


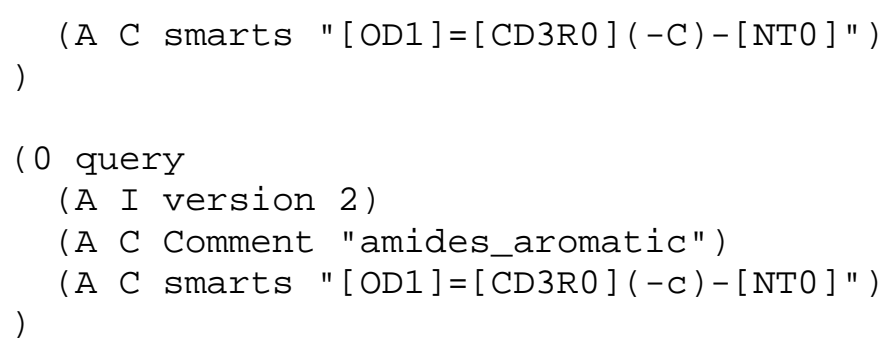

( $\odot$ Query

(A C Comment "amines_aromatic_secondary_2") 


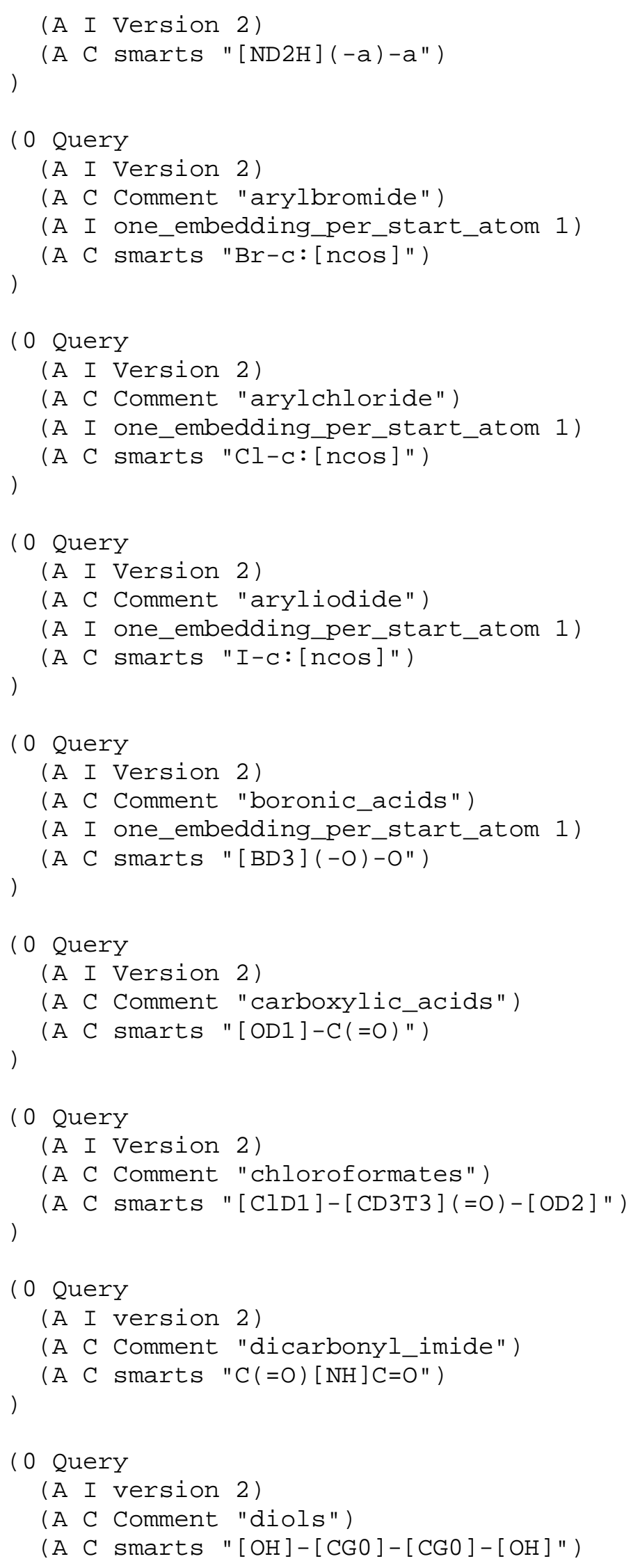




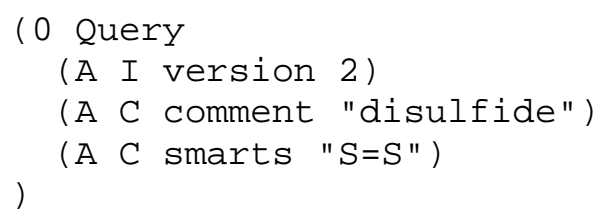

( $\odot$ Query

(A I version 2)

(A C Comment "ethers")

)

(A C smarts "[OD2ROTO](-[CGO])-[CGO]")

(०) Query

(A I version 2)

(A C Comment "fragments")

)

(A I min_number_fragments 2)

(०) Query

(A I version 2)

(A C comment "guanidines")

)

(A C smarts " $[\mathrm{ND} 1 \mathrm{H}]=[\mathrm{CD} 3 \mathrm{~T} 3](-\mathrm{N})-\mathrm{N} "$ )

( $\odot$ Query

(A C Comment "halides_alkyl")

(A I Version 2)

)

(A C smarts "[I, Br, Cl]-[CX4T1]")

(०) Query

(A C Comment "halides_aryl")

(A I Version 2)

)

(A C smarts "[I, Br, Cl;D1]-a")

( $\odot$ Query

(A C Comment "halides_benzyl")

(A I Version 2)

(A C smarts "[I, Br, Cl;D1][CH2]-C") 


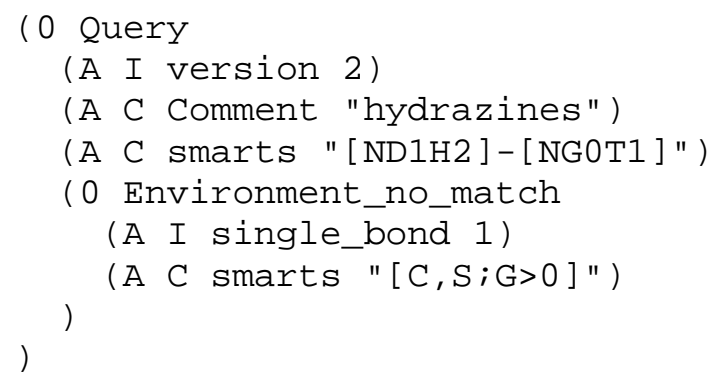

(๑) Query

(A I version 2)

(A C Comment "indole")

)

(A C smarts "[nH]1ccc2ccccc12")

( $\odot$ Query

(A C Comment "isocyanates")

(A I Version 2)

)

(A C smarts " $[\mathrm{OD} 1]=\mathrm{C}=\mathrm{N} "$ )

( $\odot$ Query

(A C Comment "isocyanides")

(A I Version 2)

(A C smarts "[N+]\#[C-]")

)

( $\odot$ Query

(A C Comment "isothiocyanates")

(A I Version 2)

)

(A C smarts " $[\mathrm{SD} 1]=\mathrm{C}=\mathrm{N} "$ ) 


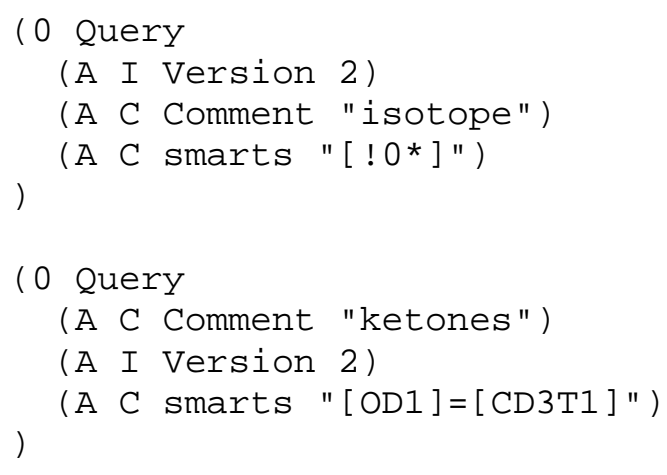

(๑) Query

(A I version 2)

(A C Comment "NNH2")

)

(A C smarts "[ND1H2]-[NGO]")

( 0 Query

(A I only_match_largest_fragment 1)

(A I Version 2)

(A C Comment "nonorganic")

)

(A C smarts "[!\#5\&!\#6\&!\#7\&!\#8\&!\#9\&!\#15\&!\#16\&!\#17\&!\#35\&!\#53\&!\#1]")

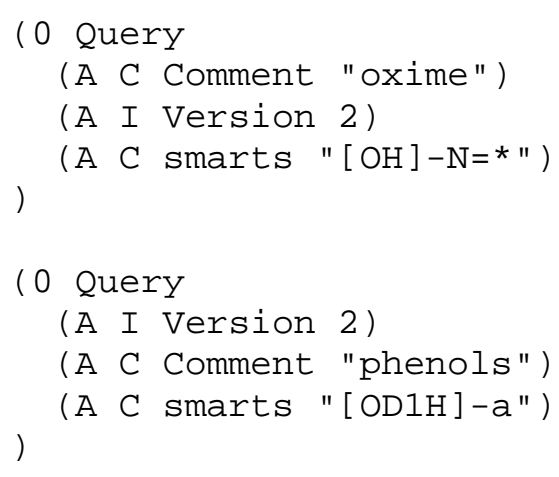




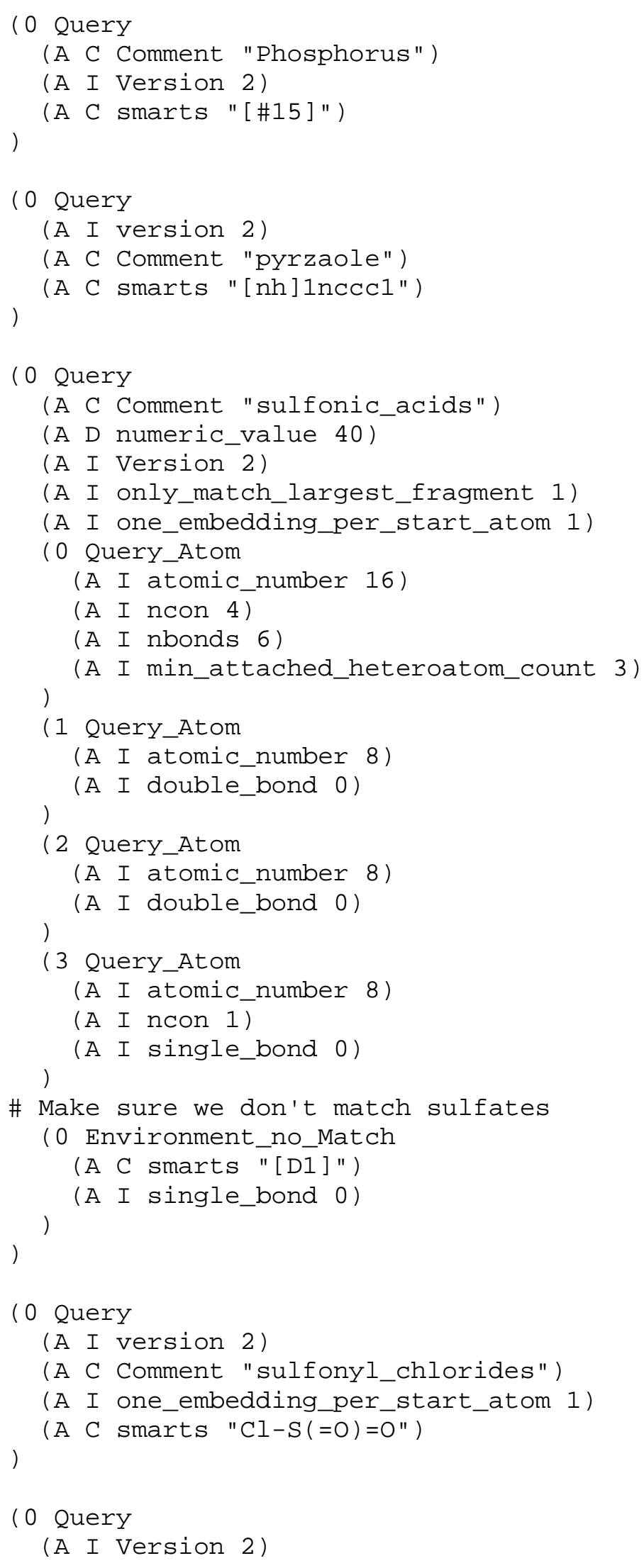




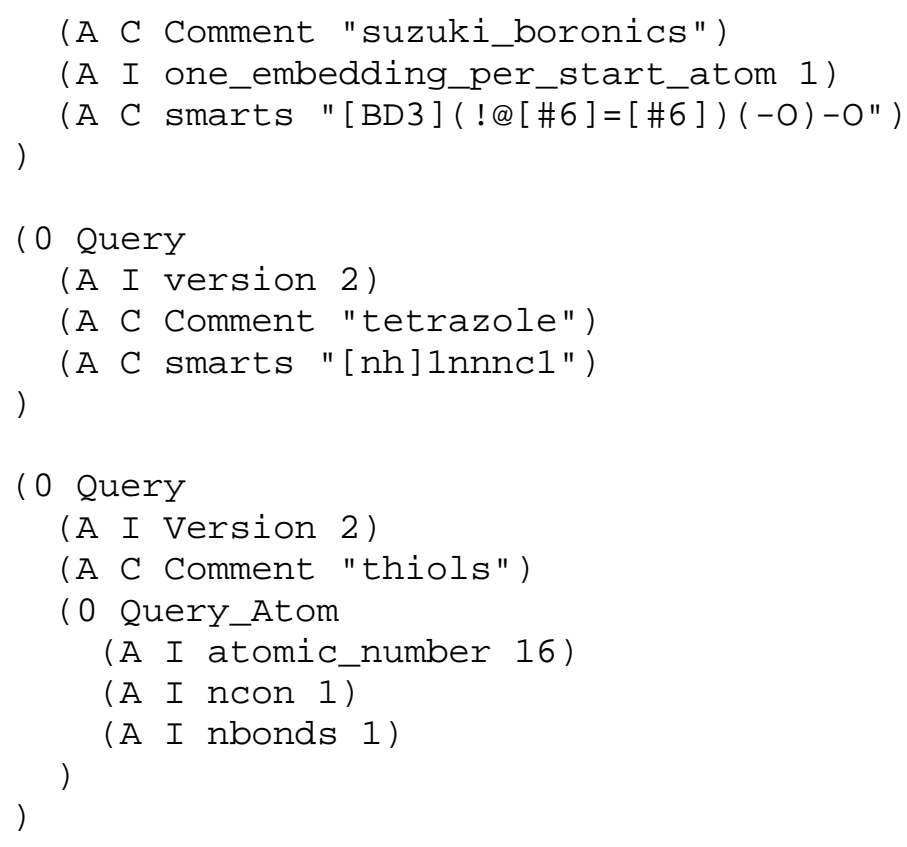




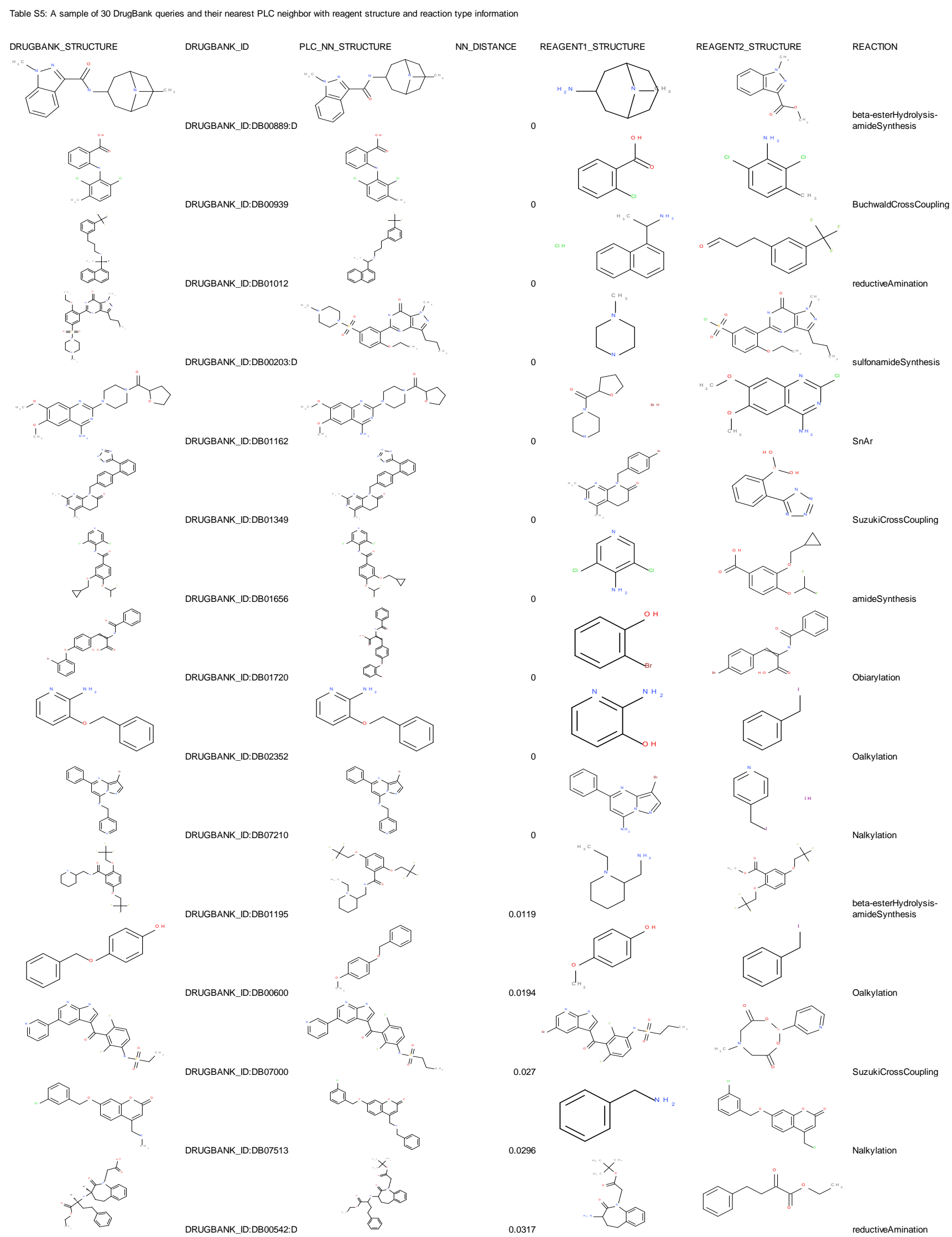




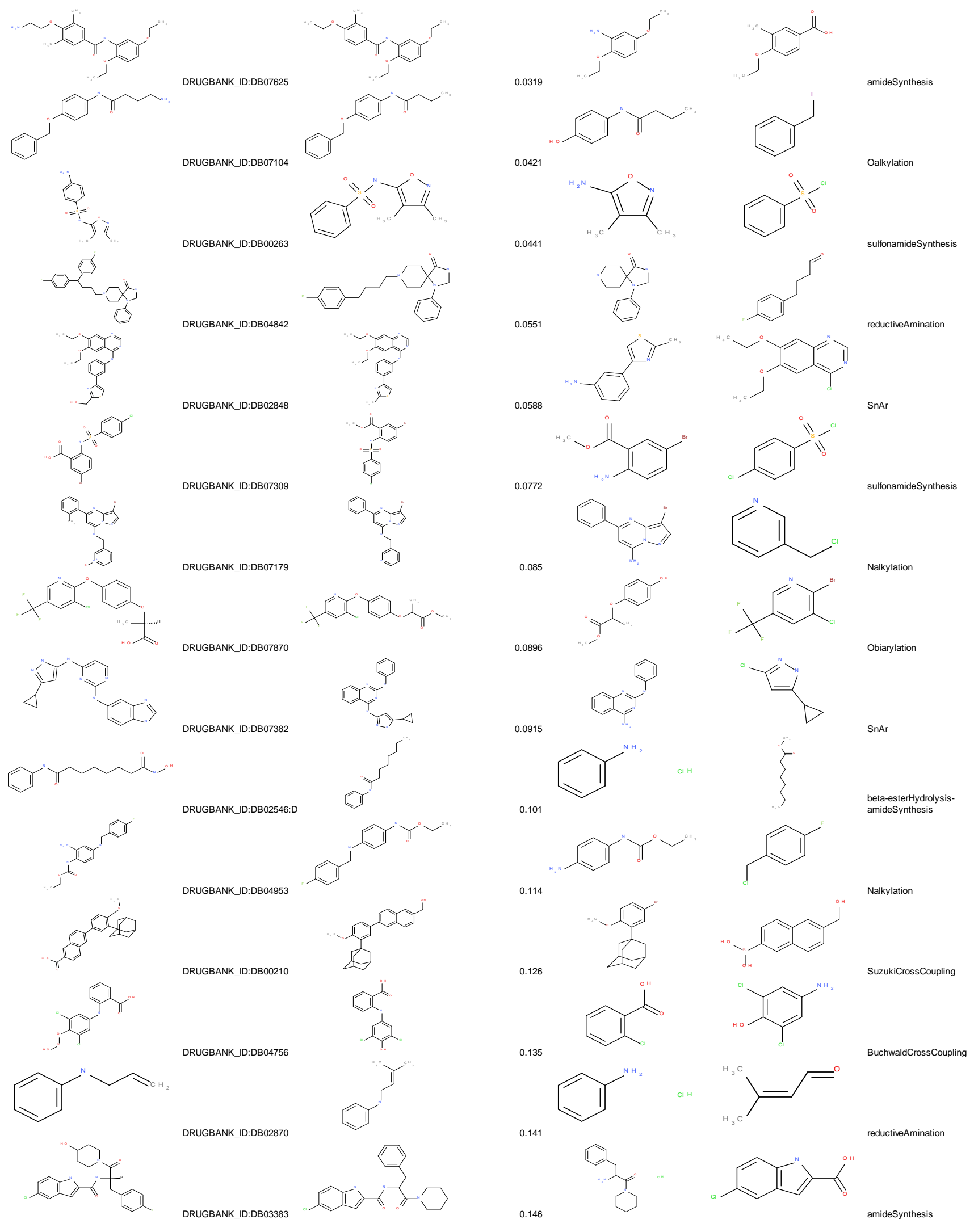


Table S6: clogP, rotatable bonds and heterotatoms property distribution of two PLC subsets generated using RSCRS and internal reagents (PLC-Subset-1), external reagents (PLC-Subset-2), a Lilly collection subset (LLY) and a Pubchem subset. All subsets have size 1million and have been filtered using the Lilly medicinal chemistry rules.
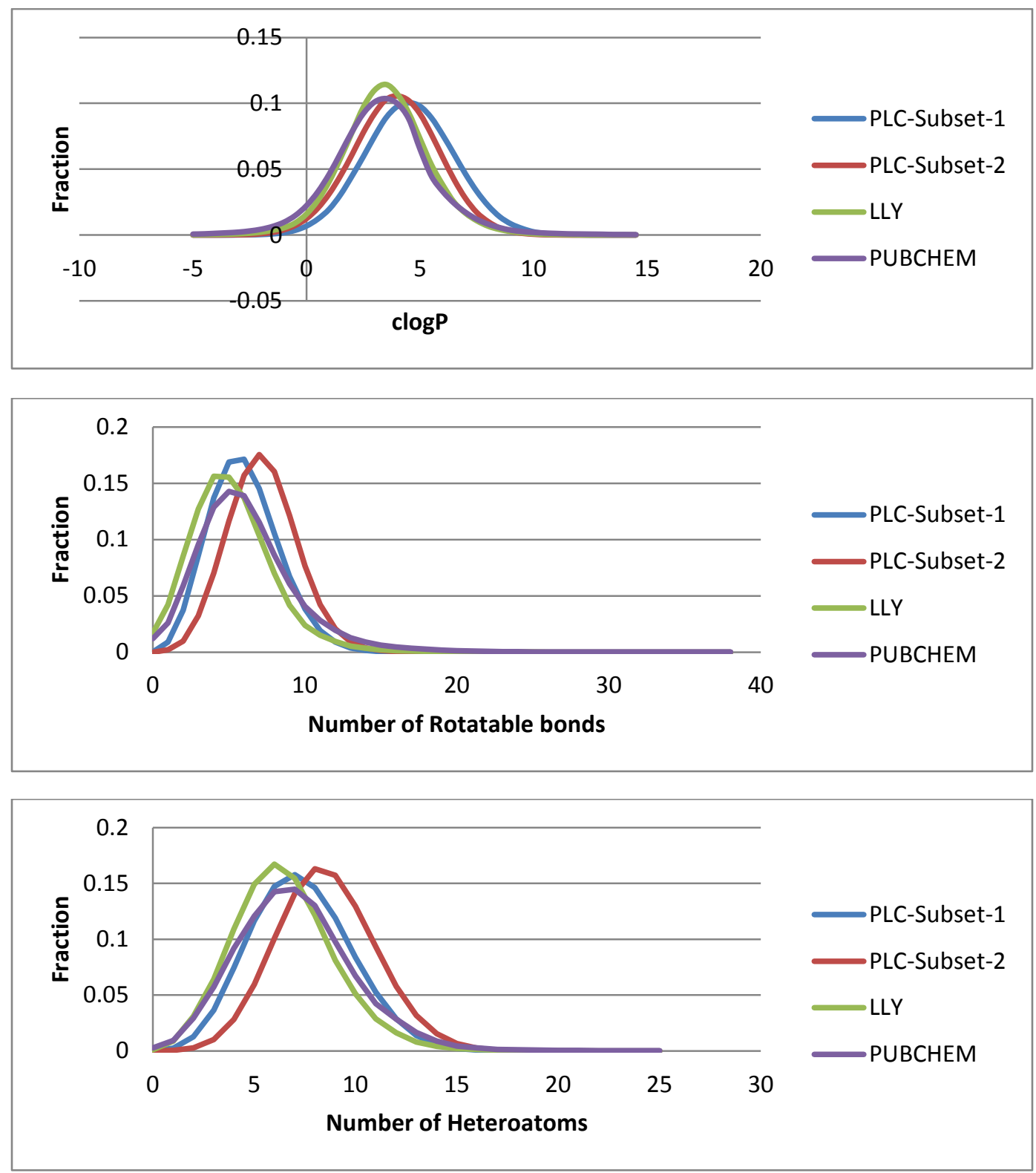